\title{
The Art of Polythene Degrading Bacteria: The Bio-Deteriorating Action of Microbial Communities
}

\section{Manas Chakraborty ${ }^{1 *}$, Indranil Chatterjee ${ }^{2 *}$, Sayak Samanta ${ }^{3}$ and Arindam Chakraborty ${ }^{3}$}

${ }^{1}$ Professor, Department of Pharmaceutical Biotechnology, Calcutta Institute of Pharmaceutical Technology and Allied Health Sciences, Howrah, West Bengal, India

${ }^{2}$ Assistant Professor, Birbhum Pharmacy School, Birbhum, West Bengal, India

${ }^{3}$ M. Pharm, Calcutta Institute of Pharmaceutical Technology and Allied Health

Sciences, Howrah, West Bengal, India

*Corresponding Author: Manas Chakraborty, Professor, Department of Pharmaceutical Biotechnology, Calcutta Institute of Pharmaceutical Technology and Allied Health Sciences, Howrah, West Bengal, India and Indranil Chatterjee, Assistant Professor, Birbhum Pharmacy School, Birbhum, West Bengal, India.
Received: June 15, 2020

Published: July 30, 2020

(C) All rights are reserved by Manas

Chakraborty, Indranil Chatterjee., et al.

\begin{abstract}
Plastic is a significant reason for Environmental Pollution. Major earth surfaces, sea waters are getting polluted day by day with accumulation of plastic as waste material from different sources which is a serious concern for human beings and different marine animals living in this world. A few stages happen in the plastic biodegradation process and could be distinguished by explicit phrasing. Bio-weakening characterizes the activity of microbial networks and other decomposer life forms answerable for the physical and synthetic decay that brought about a shallow debasement that changes the mechanical, physical and substance properties of the plastic. Bio-discontinuity alludes to the reactant activities that separate polymeric plastics into oligomers, dimers or monomers by ectocompounds or free-radicals discharged by microorganisms. Osmosis portrays to the incorporation of particles moved in the cytoplasm in the microbial digestion. Mineralisation alludes to the total corruption of atoms that brought about the discharge of totally oxidized metabolites $\left(\mathrm{CO}_{2}, \mathrm{~N}_{2}, \mathrm{CH}_{4}, \mathrm{H}_{2} \mathrm{O}\right)$. The microbial species discovered related with the debasing materials were recognized as two Gram positive and five Gram negative microscopic organisms. The microbial species related with the polythene materials were recognized as Bacillus subtilis, Bacillus amylolyticus, Bacillus cereus, Pseudomonas species etc. of which Bacillus amylolyticus causes $30 \%$ degradation and Bacillus subtilis causes $20 \%$ average degradation in a month. In this review article different isolation and identification processes followed so far for these important categories of microbes have been discussed for general awareness of research in this field.
\end{abstract}

Keywords: Biodeterioration; Biofragmentation, Assimilation; Mineralization; Pollution

\section{Introduction}

Plastics are characterized as the polymers (strong materials) which on warming become portable and can be thrown into molds
[1]. They are non-metallic pliant mixes and the materials that are produced using them can be driven into any ideal shape and sizes. Regularly plastics are utilized in numerous reasons including bun- 
dling, expendable diaper backing, rural movies and angling nets. Plastics and their utilization have become a section in all divisions of economy [2].

Framework, for example, agribusiness, media transmission, building and development, purchaser merchandise, bundling, wellbeing and clinical are generally high development territories that guarantees present interest for plastics. Plastic is the mother business to several segments and items that are produced and utilized in our everyday life like cars parts, electrical merchandise, plastic furnishings, resistance materials, agribusiness funnels, bundles and sterile products, channels and fittings, tiles and ground surface, counterfeit cowhides, containers and containers, PVC shoes and sleepers many family unit things [3]. There are two sorts of plastics: thermoplastics and thermosetting polymers. Thermoplastics are plastics that don't experience substance change in their arrangement when warmed and can be formed over and over. Thermosets are expected to have interminable sub-atomic weight. These chains are made of many rehashing atomic units, known as rehashing units, got from monomers; every polymer chain will have a few thousand rehashing units. Thermosets can dissolve and can be formed into different shapes. After they are set, they stay strong.

In the thermosetting procedure, a substance response happens which is irreversible. Vulcanization of elastic is a thermosetting procedure [4]. The polyisoprene is a cheap, marginally runny material, before warming with sulfur, yet after vulcanization the item is inflexible and non-crude. Different characterizations depend on characteristics that are significant for assembling or item plan. Plastics can likewise be arranged relying upon different physical properties, for example, thickness, high rigidity, and protection from different compound items. Plastic materials cause acute toxicity after progressive accumulation into human body if consumed for a longer duration of time in the form of PET bottles, packaged food materials, other sources of edible products which may even lead to malignancy causing damage to liver, kidney etc. resulting in mortality [5].

In this way, biodegradation in a huge scope by famous biodegrading organisms is a significant subject of examination by ecological microbiologists everywhere throughout the world. Microorganisms use polythene film as a sole wellspring of carbon bringing about fractional debasement of plastics [6]. They colonize on the outside of the polyethylene films framing a biofilm. Cell surface hydrophobicity of these living beings was seen as a significant factor in the arrangement of biofilm on the polythene surface, which subsequently improves biodegradation of the polymers. When the life forms get appended to the surface, begins developing by utilizing the polymer as the carbon source. In the essential debasement, the primary chain cuts prompting the arrangement of low-sub-atomic weight sections (oligomers), dimers or monomers [7]. The debasement is because of the extra cell protein discharged by the creature. These low sub-atomic weight mixes are additionally used by the organisms as carbon and vitality sources. The resultant breakdown parts must be totally utilized by the microorganisms, in any case there is the potential for ecological and wellbeing outcomes.

\section{Biodegradation as a sole wellspring of carbon}

Any physical or synthetic change in polymer because of natural factors, for example, light, heat, dampness, substance conditions and organic movement is named as debasement of plastic. Biodegradable polymers are intended to corrupt upon removal by the activity of living beings. Microbial corruption of plastics is brought about by enzymatic exercises that lead to a chain cleavage of the polymer into monomers. Microorganisms use polythene film as a sole wellspring of carbon bringing about fractional corruption of plastics. They colonize on the outside of the polyethylene films shaping a biofilm.

Cell surface hydrophobicity of these creatures was seen as a significant factor in the arrangement of biofilm on the polythene surface, which thusly upgrades biodegradation of the polymers. When the life forms get joined to the surface, begins developing by utilizing the polymer as the carbon source. In the essential corruption, the principle bind divides prompting the development of lowsub-atomic weight parts (oligomers), dimers or monomers. The debasement is because of the extra cell protein emitted by the life form. These low sub-atomic weight mixes are additionally used by the microorganisms as carbon and vitality sources [8]. The resultant breakdown parts must be totally utilized by the microorganisms, in any case there is the potential for ecological and wellbeing outcome. The biodegradation of plastics and related polymers by various classifications of microorganisms could assume a significant job in observing of ecological contamination uncommonly in marine condition sparing an immense populace of marine animals from early demise. 
Screening and isolation of plastic degrading microbes from different sources

Different methods of screening and isolation of microbes are followed for plastic degrading bacteria. These types of bacteria include different species of Bacillus, Pseudomonas, Agrobacterium which show different degrees of deterioration when applied on plastics and other synthetic polymers. For isolation of soil microbes, soil samples from different dumped areas are collected, washed properly and enriched soil sample is prepared. From this enriched soil sample serial dilution is made and inoculated in nutrient agar media along with stripes of polythene or plastic sheets and incubated for 24 - 48 hours. After proper incubation the type of colonies are identified by gram staining method. After morphological identification different biochemical tests are performed for confirmation of nature of strain isolated from soil sources:

\section{Catalase test}

The catalase test is performed to distinguish the nearness of catalase chemical by immunizing a loopful of culture into tubes containing $3 \%$ of hydrogen peroxide arrangement. Positive outcome is demonstrated by arrangement of fizz or presence of air pockets because of the separating of hydrogen peroxide to $\mathrm{O}_{2}$ and $\mathrm{H}_{2} \mathrm{O}$ [9].

\section{Mannitol test}

This investigation is by and large performed to decide if the microbes is fit for maturing mannitol sugar or not. At whatever point life form mature mannitol sugar, the $\mathrm{pH}$ of the media gets acidic because of the creation of acids. The aging of the media from red to yellow show positive outcome [10].

\section{Motility test}

The motility test is done to decide the motility of the creature. Around $5 \mathrm{ml}$ of the supplement stock was vaccinated with the confined microorganism and was brooded for a short-term development. At that point a drop of the NB media was taken on a scored slide (hanging drop technique) and was seen under stage differentiate magnifying instrument [10].

\section{Citrate test}

This test decides the capacity of the microbes to change over citrate into oxaloacetate. Citrate is the main carbon source accessible to the microscopic organisms in this strategy. Positive outcomes are checked whether the microbes develop and the media transforms into splendid blue shading [11].

\section{Luminescence production}

One plate each of the two isolated strain are kept under U.V meter and checked for the production of any bioluminescence.

\section{Alternate method of isolation}

In this method the collected soil samples are inoculated in liquid broth, incubated for 24 - 48 hours for growth of microbes. Then one loopful of liquid broth is taken, streaked on prepared agar plate and proper colony isolated, counted and identified by gram staining method. Mostly the colony respond to gram positive type. Now the isolated microbes are inoculated in liquid broth along with stripes of plastics/PVC strips and rotated in shaker incubator for 30 - 45 days at 110 - $120 \mathrm{rpm}$ at 370 - 400 Celsius. After that the stripes are collected, washed with distilled water, dried and checked for loss of weight based on initial weight of stripes [12].

During static incubation after six weeks period, biofilms are produced by specific categories of microbes. However, after that the inoculating media becomes dry due to rapid dehydration and no further isolation is possible.

Plastics isolated from cold marine environment which causes pollution in deep seas causing damage/death to large no of rare marine inhabitants, living in extreme conditions can also be biodegraded by specific microbial isolates surviving also in extreme cold conditions.

Degree of weight loss for different categories of plastics/PVC by different microbes

By scrutiny of different literature in connection with the review following observations are made [6-12] (Table).

Hydrolase enzymes from Bacillus sp. BCBT21 were detected at $55^{\circ} \mathrm{C}$ during the treatment. These enzymes were extracellular including CMCase, protease, chitinase, xylanase, and lipase with low activity and the activities were decreased over the treatment time. High temperature also takes part in the plastic degrading process as a thermal factor. Apart from these other bacterial and fungal species also take active part in degradation of different categories of plastics from different sources. 


\begin{tabular}{|l|c|c|c|c|}
\hline SI No & Bacteria Type & Plastics Type & Incubation Period & Deterioration (\%) \\
\hline 1 & Bacillus subtilis & HDPE/LDPE & $30-45$ days & $20 \%$ (approx.) \\
\hline 2 & Bacillus cereus & HDPE/LDPE/PET & 45 days & $25-30 \%$ \\
\hline 3 & Pseudomonas sp & Natural Polythene & 45 days & $46-47 \%$ \\
\hline 4 & Bacillus sp BCBT21 & Natural Plastics & 30 days & $60-61 \%$ \\
\hline 5 & Bacillus amylolyticus & Different polymers & 30 days in liquid shake culture & $40-50 \%$ \\
\hline
\end{tabular}

Table

Ideonella sakaiensis was first distinguished in 2016 by a group of specialists drove by Kohei Oda of Kyoto Institute of Technology and Kenji Miyamoto of Keio University in the wake of gathering an example of PET-tainted dregs close to a plastic container reusing office in Japan. Ideonella sakaiensis is a bacterium from the variety Ideonella and family Comamonadaceae equipped for separating and expending the plastic poly (ethylene terephthalate) (PET) as a sole carbon and vitality source. Researchers have made a freak protein that separates plastic beverages bottles - unintentionally. The advancement could help illuminate the worldwide plastic contamination emergency by empowering just because the full reusing of containers [12].

\section{Discussion}

From the above review, it is seen that microbial degradation plays an important role in rapid degradation of plastics/PVC material after stipulated period of incubation or rotation in shaker incubator, probably due to enzymatic hydrolysis by microbes. The production of biofilm over PVC surface enable the microbes to utilize non soluble substrates by enzymatic degradation activities. The duration of the microbial colonization is an important factor that effects total degradation period. The process of biodegradation depends on types and incubation period of microbes which in turn regulates enzyme release and formation of biofilm. Pre-treatment of polyethylene with substances that are earth well-disposed could likewise be embraced as a way to upgrade polyethylene biodegradation by receiving the innovation a significant danger to ecological contamination could be expelled for better living of human culture. Recent advancement in identification and exploration of new genera of microbes capable of degrading polythene or plastic surface as identified in Japan has also been reflected in this review.
The $\%$ of degradation are recorded to be very significant in some species of Bacillus and Pseudomonas. The incubation period plays a major role in detecting degradation in different grades of plastic/ PVC materials.

\section{Conclusion}

Different species of microbes show different patterns of degradation under laboratory condition in specific temperature. The environmental toxicity produced by plastic/PVC material both in land and marine environment are causing health hazards to a large no of population including marine life all over the world and it is a serious concern for scientists working in this field. So, it is expected that large scale application of biodegradation process could find a better means to remove land and marine pollution caused by these daily consumable materials. Recycling of these materials are nowadays followed but more public awareness of use of proper category of reusable PVC material could throw a light on this major issue. Bacillus and Pseudomonas are very common genera which could be successfully cultivated under proper laboratory conditions to treat these plastic/PVC materials for enzymatic hydrolysis process. Future research can explore more newer species of bacteria/fungus as identified recently in Japan which would enable commercial expansion of technology to serve more human welfare purpose in different parts of world for healthy living.

\section{Acknowledgements}

We would like to express a very great appreciation to the staff associates of Department of Pharmaceutical Biotechnology, Calcutta Institute of Pharmaceutical Technology and Allied Health Sciences, Howrah, West Bengal, India.

\section{Conflict of Interest}

The authors declare no financial or any conflict of interest. 


\section{Bibliography}

1. Albertsson AC. "The shape of the biodegradation curve for low and high density polyethylenes in prolonged series of experiments". European Polymer Journal 16 (1980): 623-630.

2. Albertsson AC and Karlsson S. "The influence of biotic and abiotic environments on the degradation of polyethylene". Progress in Polymer Science 15.2 (1990): 177-192.

3. Bollag WB., et al. "Biodegradation and encyclopedia of microbiology". In Lederberg, J (ed.). Academic, New York. (2000): 461-471.

4. Cruz-Pinto JJC., et al. "The kinetics and mechanism of polyethylene photo-oxidation". Angewandte Makromolekulare Chemie 216 (1994): 113-133.

5. Huang, J., et al. "Biodegradable plastics: a review". Advances in Polymer Technology 10.1 (1990): 23-30.

6. Luzier WD. "Materials derived from biomass/biodegradable materials". Proceedings of the National Academy of Sciences of the United States of America 89.3 (1992): 839-842.

7. Mueller RJ. "Biological degradation of synthetic polyestersenzymes as potential catalysts for polyester recycling". Process Biochemistry 41.10 (2006): 2124-2128.

8. Nakayama A., et al. "Synthesis and biodegradation of poly $(\gamma$ butyrolactone-co-L-lactide)". Journal of Environmental Polymer Degradation 4 (1996): 205-211.

9. Olayan HB., et al. "Photochemical and thermal crosslinking of polymers". Journal of Macromolecular Science - Reviews in Macromolecular Chemistry and Physics 36.4 (1996): 671-719.

10. Rivard C., et al. "Starch esters as biodegradable plastics: Effects of ester group chain length and degree of substitution on anaerobic biodegradation". Enzyme and Microbial Technology 17.9 (1995): 848-852.

11. Sang BI., et al. "Fungal contribution to in situ biodegradation of poly(3-hydroxybutyrate-co-3-hydroxyvalerate) film in soil". Applied Microbiology and Biotechnology 58.2 (2002): 241-247.

12. Scott G. "Photo-biodegradable plastics: their role in the protection of the environment". Polymer Degradation and Stability 29.1 (1990): 135-154.

\section{Assets from publication with us}

- Prompt Acknowledgement after receiving the article

- Thorough Double blinded peer review

- Rapid Publication

- Issue of Publication Certificate

- High visibility of your Published work

Website: www.actascientific.com/

Submit Article: www.actascientific.com/submission.php

Email us: editor@actascientific.com

Contact us: +919182824667 